\title{
Coeficiente do tanque Classe A obtido por diferentes métodos em ambiente protegido e no campo
}

\section{Calculation of the Class-A pan coefficient in greenhouse and field by different methods}

\author{
Antonio Ribeiro Cunha ${ }^{1 *}$
}

\section{Resumo}

\begin{abstract}
Atualmente existem vários métodos para a determinação do coeficiente do tanque Classe $\mathrm{A}(\mathrm{Kp})$ na estimativa da evapotranspiração de referência (ETo), o qual é de importância para o manejo da água na agricultura. A maioria desses métodos leva em conta a velocidade do vento, a umidade relativa do ar e a área de bordadura. Este estudo foi realizado na região de Botucatu, SP, em condições de ambiente protegido e em campo nos meses de julho-agosto (seco) e setembro-outubro (chuvoso), utilizando os métodos de Doorenbos e Pruitt (1977), Cuenca (1989), Snyder (1992), Pereira et al. (1995), Allen et al. (1998) e correlação entre ETo determinada por Penman-Monteith (EToPM) e a evaporação do tanque Classe A (ECA). A EToPM foi utilizada como padrão para as correlações com a evapotranspiração de referência pelo método do tanque Classe A (EToTCA) obtida pelos diferentes métodos de Kp. As variações de Kp no ambiente protegido foram de 0,51 a 0,85, e em campo de 0,33 a 0,85. Em ambiente protegido, os métodos de Allen et al. (1998) e Snyder (1992) são os mais recomendados para meses secos, e os da correlação entre EToPM e ECA e Cuenca (1989) para meses chuvosos. Já em condição de campo, os métodos de Allen et al. (1998) e da correlação entre EToPM e ECA para os meses secos, e os de Allen et al.(1998) e Cuenca (1989) para os meses chuvosos. O método de Allen et al. (1998) foi o mais eficiente, independente do ambiente e dos meses analisados. O valor fixo de Kp só deve ser utilizado quando não houver a possibilidade de sua determinação a partir de métodos mais sofisticados. Palavras-chave: Evapotranspiração. Manejo da irrigação. Estufa agrícola.
\end{abstract}

\begin{abstract}
There are several methods for determining the Class-A pan coefficient $(\mathrm{Kp})$ to estimate the reference evapotranspiration (ETo), which is of great importance to water management in agriculture. Most of these methods take into account the wind speed, relative humidity and the fetch area. This study was done in region of Botucatu, SP, in greenhouse and field in the months of July-August (dry) and September-October (rainy), used the methods of Doorenbos e Pruitt (1977), Cuenca (1989), Snyder (1992), Pereira et al. (1995), Allen et al. (1998) and correlation between ETo determined by PenmanMonteith (EToPM) and evaporation of the Class-A pan (ECA). The EToPM was used as standard for the correlations between the ETo determined by the Class-A pan (EToTCA) obtained by different methods of $\mathrm{Kp}$. The $\mathrm{Kp}$ in greenhouse ranged between 0.51 and 0.85 , and in the field from 0.33 to 0.85. The methods of Allen et al. (1998) and Snyder (1992) are the most recommended for dry months in greenhouse and rainy months the correlation between the ECA and EToPM e Cuenca (1989). In the field condition, the methods of Allen et al. (1998) and of correlation between the ECA and EToPM for dry months, and for rainy months of Allen et al. (1998) and Cuenca (1989). The method of Allen et al. (1998) was the most efficient, regardless of the environment and of months analyzed. The fixed value of $\mathrm{Kp}$ should only be used when it is not possible to determine from its more sophisticated methods.

Key words: Evapotranspiration. Irrigation management. Greenhouse.
\end{abstract}

1 Eng ${ }^{\circ}$. Agr ${ }^{\circ}$. Dr. e Pós-Doutorado em Produção Vegetal. Departamento de Recursos Naturais. Faculdade de Ciências Agronômicas, Universidade Estadual Paulista Júlio de Mesquita Filho, Botucatu, SP. E-mail: arcunha@fca.unesp.br

* Autor para correspondência

Recebido para publicação 18/10/2009 Aprovado em 15/03/2011 


\section{Introdução}

Aágua doce é um recurso escasso e irregularmente distribuído pelo globo terrestre. Do total de água disponível no mundo, apenas $2,5 \%$ são de água doce e somente $0,007 \%$ desse valor é acessível ao consumo, sendo que a agricultura utiliza 70\% desses 0,007\% para a produção de alimentos (WRI, 2009). Esta produção está condicionada a fatores ambientais que, em muitos casos, prejudicam o desenvolvimento das plantas, obrigando determinadas regiões a utilizar ambientes protegidos. Esses ambientes causam modificações físicas nos diferentes elementos meteorológicos, as quais ainda não são totalmente conhecidas, sobretudo, no que se refere ao manejo de água.

Entre os elementos meteorológicos que sofrem modificações importantes no interior do ambiente protegido está a evaporação, que conseqüentemente, altera também a evapotranspiração. A demanda evaporativa do ar no interior de ambiente protegido é menor do que em condição externa, devido principalmente à atenuação da radiação solar e diminuição da velocidade do vento, segundo resultados de pesquisas desenvolvidas por Prados (1986), Fernandez, Orgaz e Lopez-Gálvez (1995), Alves e Klar (1996), Martins et al. (1999), Braga e Klar (2000), Buriol et al. (2001), Cunha (2001), Cunha, Escobedo e Klosowski (2002), Fernandes, Corá e Araújo (2004), Rezende et al. (2004) e Vásquez et al. (2005). Esta menor demanda varia de acordo com as condições meteorológicas predominantemente de cada dia, local e/ou região, sendo em geral, de 60 a $80 \%$ menor em relação à condição externa (VAN DER POST; VAN-SHIE; GRAAF, 1974; MONTERO et al., 1985; PRADOS, 1986; ROSENBERG; McKENNY; MARTIN, 1989; FARIAS, 1991; STANGHELLINI, 1993; FARIAS; BERGAMASCHI; MARTINS, 1994; MARTINS; GONZALEZ, 1995; SENTELHAS, 2001; STAMATO JUNIOR, 2007).

A evapotranspiração de referência (ETo) em ambiente protegido pode ser estimada por vários métodos, sendo o de Penman-Monteith recomendado pela FAO-56 (ALLEN et al., 1998). Outro método recomendado pela FAO-56 é o método do tanque Classe A, que apresenta facilidade no manejo e acuracidade nas medidas obtidas, sendo atualmente muito usado no Brasil. Trata-se de um método bastante simples, com custo baixo em relação aos equipamentos necessários para a estimativa da ETo a partir de métodos combinados, e é bastante difundido nas áreas irrigadas para estimativa da necessidade de irrigação das culturas.

O método do tanque Classe A (TCA) é baseado no princípio de que a água contida no seu interior não deve oferecer nenhum impedimento ao processo evaporativo. A evaporação obtida neste tanque será sempre maior em relação à perda efetiva de uma cultura, mesmo estando ela em condições ótimas de suprimento de água. Assim, a determinação da evapotranspiração de referência pelo método do TCA depende de dois coeficientes de correção, o do tanque (Kp) que é função da velocidade do vento, da umidade relativa do ar e do tamanho da bordadura que o circunda, e o da cultura $(\mathrm{Kc})$ que envolve as características físicas do solo e a fisiologia da planta.

Atualmente, existem diversas metodologias para a obtenção do $\mathrm{Kp}$, as quais podem resultar em valores diferentes dependendo do local e das condições climáticas (ALLEN et al., 1998), e com isso, Chiew, Kamaladasa e Malano (1995) somente recomendam a estimativa da EToTCA quando o Kp é bem determinado, recomendando sua obtenção por meio da correlação entre EToPM e ECA.

Considerando que as coberturas plásticas propiciam condições ambientais bastante diferentes daquelas encontradas em campo, e que são impermeáveis aos líquidos, impedindo a entrada da precipitação pluvial, torna-se importante um melhor conhecimento das exigências hídricas das plantas no interior de ambientes protegidos, uma vez que a irrigação será a única forma de suprimento de água às culturas. Além disso, com o aumento da utilização do ambiente protegido no Brasil, faz-se 
necessário a geração de novas tecnologias a respeito do manejo desse ambiente.

Neste contexto, o trabalho teve por objetivo avaliar diferentes métodos de determinação do $\mathrm{Kp}$ em ambiente protegido e em campo no município de Botucatu, SP, e analisar a sua influência na estimativa da ETo pelo método do tanque Classe A comparativamente ao método de Penman-Monteith em escala diária.

\section{Material e Métodos}

O período usado nesta análise foi de 01/07/99 à $31 / 10 / 99$, em escala diária, sendo dois meses secos (Jul-Ago) e dois meses chuvosos (Set-Out), obtidos na área experimental próxima $(200 \mathrm{~m})$ à estação meteorológica do Departamento de Recursos Naturais da Faculdade de Ciências Agronômicas da Universidade Estadual Paulista, Campus de Botucatu, SP, (latitude de $22^{\circ} 51^{\prime} \mathrm{S}$; longitude de $48^{\circ}$ 26'W e altitude de 786 m). Segundo a classificação climática de Köppen, o clima é $C f a$, clima temperado quente (mesotérmico) úmido, e a temperatura média do mês mais quente é superior a $22{ }^{\circ} \mathrm{C}$; e segundo a classificação de Thornthwaite é $\mathrm{B}_{2} \mathrm{rB}_{3}{ }_{3} \mathrm{a}^{\prime}$ (clima úmido com pequena deficiência hídrica - abril, julho e agosto, mesotérmico, com evapotranspiração potencial anual de $945,15 \mathrm{~mm}$ e concentração da evapotranspiração potencial no verão igual a 33\%) (CUNHA; MARTINS, 2009).

O ambiente protegido utilizado foi do tipo túnel alto não-climatizado e orientado predominantemente no sentido N-S, constituído de estrutura de ferro galvanizado e coberto com polietileno transparente de $120 \mu \mathrm{m}$ e tela plástica preta fixa nas laterais, com $50 \%$ de transparência para a radiação solar (sombrite). A casa de vegetação apresenta 7,0 m de largura por 40,0 $\mathrm{m}$ de comprimento, altura do pé direito de 2,2 $\mathrm{m}$ e do arco central de 4,0 m, acima do nível do solo. À campo, utilizou dados meteorológicos obtidos em estação meteorológica automática da Faculdade de Ciências Agronômicas.

A evaporação em tanque Classe A (ECA) foi quantificada em ambiente protegido e em estação meteorológica. No ambiente protegido, o tanque foi instalado no centro da estufa agrícola, sendo que as leituras de ambos os tanques eram realizadas diariamente às 9:00 h para comparação.

Os dados meteorológicos coletados foram de temperatura e umidade relativa do ar a $2 \mathrm{~m}$ altura (sensor Vaisala modelo HMP45C), velocidade do vento (sensor R.M. Young modelo 031015), pressão atmosférica (sensor Vaisala modelo PTB101B), saldo de radiação (sensor REBS modelo Q7.1) e fluxo de calor no solo (sensor REBS modelo HTF-3). A coleta desses sensores automatizados foi feita por meio de um sistema de aquisição de dados (Micrologger 21X), tanto em ambiente protegido como na estação meteorológica. A varredura dos sensores era realizada a cada 5 segundos, com saída média dos dados a cada 5 minutos.

Para o cálculo do $\mathrm{Kp}$ em escala diária foram utilizados cinco métodos:

\section{1) Método de Doorenbos e Pruitt (1977)}

Obtido segundo a tabela de Doorenbos e Pruitt (1977), em função da velocidade do vento à $2 \mathrm{~m}$ altura $(\mathrm{km} / \mathrm{d})$, umidade relativa do ar $(\%)$ e bordadura $(\mathrm{m})$, sendo que para o ambiente protegido foi de $10 \mathrm{~m}$ de cobertura verde e de $30 \mathrm{~m}$ para a estação meteorológica.

\section{2) Método de Cuenca (1989)}

$$
K p=0,475-2,4 \cdot 10^{-4} \cdot V+5,1610^{3} \cdot U R+1,1810^{3} \cdot B-16 \cdot 10^{5} \cdot U R^{2}-1.1 \sigma^{6} B^{2}-8 \cdot 10^{9} \cdot U R^{2} \cdot V-1.10^{-8} \cdot U R^{2} B
$$

em que $\mathrm{V}$ é a velocidade do vento a $2 \mathrm{~m}$ altura ( $\mathrm{km} /$ dia), UR a umidade relativa do ar (\%) e B a bordadura com vegetação em torno do tanque (m). 
3) Método de Snyder (1992)

$K p=0,482+0,024 \ln (B)-0,000376 . V+0,0045 . U R$

4) Método de Pereira et al. (1995)

$K p=\frac{0,85(s+\gamma)}{(s+\gamma)(1+0,34 . V)}$

em que $\mathrm{V}$ a velocidade do vento à $2 \mathrm{~m}$ de altura $(\mathrm{m} / \mathrm{s})$, s é a declividade da curva de pressão de vapor $\left(\mathrm{kPa} /{ }^{\circ} \mathrm{C}\right)$ calculada pela expressão:

$s=\frac{4098 \cdot e_{s}}{(T+237,3)^{2}}$

em que T é a temperatura média do ar $\left({ }^{\circ} \mathrm{C}\right)$, es a pressão de saturação $(\mathrm{kPa})$ calculada pela expressão:

$e_{s}=0,6108 \cdot 10^{\left(\frac{7,5 . T}{T+237,3}\right)}$

e $\gamma$ o coeficiente psicrométrico igual a $0,0725 \mathrm{kPa} /{ }^{\circ} \mathrm{C}$, para uma pressão atmosférica média de $90,66 \mathrm{kPa}$ e constante psicrométrica de $80.10^{-5 / 0} \mathrm{C}$.

5) Método de Allen et al. (1998)

$K p=0,108-0,0286 \cdot V+0,0422 \cdot \ln (B)+0,1434 \cdot \ln (U R)-0,000631[\ln (B)]^{2} \cdot \ln (U R)$

em que $\mathrm{V}$ é a velocidade do vento a $2 \mathrm{~m}$ altura $(\mathrm{m} / \mathrm{s})$, UR a umidade relativa do ar $(\%)$ e B a bordadura com vegetação em torno do tanque $(\mathrm{m})$.

\section{6) Método da correlação entre EToPM e ECA}

$$
E T o P M=f(E C A)
$$

Os valores de Kp foram obtidos considerando o período seco (julho e agosto) e chuvoso (setembro e outubro). Ambos os períodos analisados são importantes em ambiente protegido, sendo que o período seco normalmente indica uma maior necessidade de irrigação. Assim, a ETo pelo método TCA foi obtida pela expressão:

$$
\text { EToTCA }=K p(E C A)
$$

A ETo diária foi determinada pelo método de Penman-Monteith (ALLEN et al., 1998), considerada padrão neste estudo para o cálculo de Kp, segundo a FAO-56:

$$
\text { EToPM }=\frac{0,408 . s(R n-G)+\gamma\left(\frac{900}{T+273}\right) U_{2}\left(e_{s}-e_{a}\right)}{s+\gamma\left(1+0,34 U_{2}\right)}
$$

em que Rn é o saldo de radiação ( $\left.\mathrm{MJ} / \mathrm{m}^{2} / \mathrm{dia}\right)$; G o fluxo de calor no solo $\left(\mathrm{MJ} / \mathrm{m}^{2} / \mathrm{dia}\right)$; $\gamma$ o coeficiente psicrométrico igual a $0,0725 \mathrm{kPa} /{ }^{\circ} \mathrm{C} ; \mathrm{T}$ a temperatura média do $\operatorname{ar}\left({ }^{\circ} \mathrm{C}\right) ; U_{2}$ a velocidade média do vento a $2 \mathrm{~m}$ de altura (m/s). A pressão de saturação $(e, \mathrm{kPa})$ foi calculada pela expressão:

$e_{s}=0,6108 \cdot 10^{\left(\frac{7,5 . T}{T+237,3}\right)}$ 
em que T é a temperatura média do ar $\left({ }^{\circ} \mathrm{C}\right)$.

A pressão atual de vapor $\left(e_{a}, \mathrm{kPa}\right)$ foi calculada pela expressão:

$$
e_{a}=\frac{e_{s} \cdot U R}{100}
$$

em que UR é a umidade relativa do ar (\%).

A declividade da curva de pressão de vapor $\left(s, \mathrm{kPa}^{\circ} \mathrm{C}^{-1}\right)$ foi determinada pela expressão:

$$
s=\frac{4098 \cdot e_{s}}{(T+237,3)^{2}}
$$

em que $\mathrm{T}$ é a temperatura média do ar $\left({ }^{\circ} \mathrm{C}\right)$.

As comparações entre os métodos de cálculo de $\mathrm{Kp}$ foram feitas por meio dos seguintes indicadores estatísticos: análise de regressão - coeficiente de determinação $\left(\mathrm{R}^{2}\right)$, índice de concordância (d), erro máximo absoluto (ME), erro médio absoluto (MBE) e eficiência (EF), segundo Willmott et al. (1985):

$d=1-\left[\frac{\sum_{i=1}^{n}\left(O_{i}-E_{i}\right)^{2}}{\sum_{i=1}^{n}\left(\left|O_{i}-\bar{E}\right|+\left|E_{i}-\bar{E}\right|\right)^{2}}\right]$

$M E=\max \left(\left|E_{i}-O_{i}\right|\right)_{i=1}^{n}$

$M B E=\frac{1}{n} \sum_{i=1}^{n}\left(E_{i}-O_{i}\right)$

$$
E F=\frac{\sum_{i=1}^{n}\left(O_{i}-\bar{O}\right)^{2}-\sum_{i=1}^{n}\left(O_{i}-E_{i}\right)^{2}}{\sum_{i=1}^{n}\left(O_{i}-\bar{O}\right)^{2}}
$$

em que $E i$ é a evapotranspiração de referência diária obtida pelo tanque Classe A através dos diferentes métodos, $O_{i}$ a evapotranspiração de referência diária determinada pelo método Penman-Monteith, $\overline{\mathrm{E}}$ a média evapotranspiração de referência diária obtida pelo tanque Classe A através dos diferentes métodos, $\overline{\mathrm{O}}$ a média da evapotranspiração de referência diária obtida pelo método Penman-Monteith, e $n$ o número de observações.

\section{Resultados e Discussão}

$\mathrm{Na}$ (Figura 1) são apresentados os valores de Kp em função da correlação entre evapotranspiração de referência de Penman-Monteith (EToPM) e a evaporação do tanque Classe A (ECA), para o ambiente protegido de 0,62 e 0,57 , e para a condição de campo de 0,68 e 0,61, respectivamente para os meses de Jul-Ago e Set-Out. A condição de ambiente 
protegido apresentou um menor $\mathrm{Kp}$, com uma dados em condição de campo; e valores de ECA evaporação de 27 a 34\% menor, e consequentemente, e ETo nos meses de Set-Out tendendo a zero para uma evapotranspiração 32 a 39\% menor nos meses os dois ambientes, indicando dias com chuva e/ou analisados. Houve uma maior variabilidade dos nebulosidade (Figura 1 e Tabela 1).

Tabela 1. Valores médios dos elementos meteorológicos medidos e da evapotranspiração de referência por Penman-Monteith (EToPM). T = temperatura do ar; $\mathrm{UR}=$ umidade relativa do ar; $\mathrm{V}=$ velocidade do vento; $\mathrm{Rn}=$ saldo de radiação; ECA = evaporação do tanque Classe A. Botucatu, SP.

\begin{tabular}{llllccc}
\hline Meses (ambiente) & $\mathbf{T}^{\left({ }^{\circ} \mathbf{C}\right)}$ & $\mathbf{U R}(\%)$ & $\mathbf{V}(\mathbf{k m} / \mathbf{d})$ & $\mathbf{R n}\left(\mathbf{M J} / \mathbf{m}^{2}\right)$ & $\mathbf{E C A}(\mathbf{m m})$ & $\mathbf{E T o P M}(\mathbf{m m})$ \\
\hline Jul-Ago (pt) & 19,6 & 61,4 & 7,4 & 7,2 & 3,1 & 2,0 \\
Jul-Ago (ca) & 19,0 & 62,0 & 175,1 & 9,3 & 4,7 & 3,3 \\
Set-Out (pt) & 20,6 & 64,9 & 9,9 & 8,6 & 4,3 & 2,5 \\
Set-Out (ca) & 19,9 & 65,8 & 226,5 & 10,9 & 5,9 & 3,7 \\
\hline
\end{tabular}

*Dados medidos no período de 01/07/99 à 31/10/99 obtidos na área experimental próxima (200 m) à estação meteorológica do Departamento de Recursos Naturais da Faculdade de Ciências Agronômicas da Universidade Estadual Paulista, Campus de Botucatu, SP.
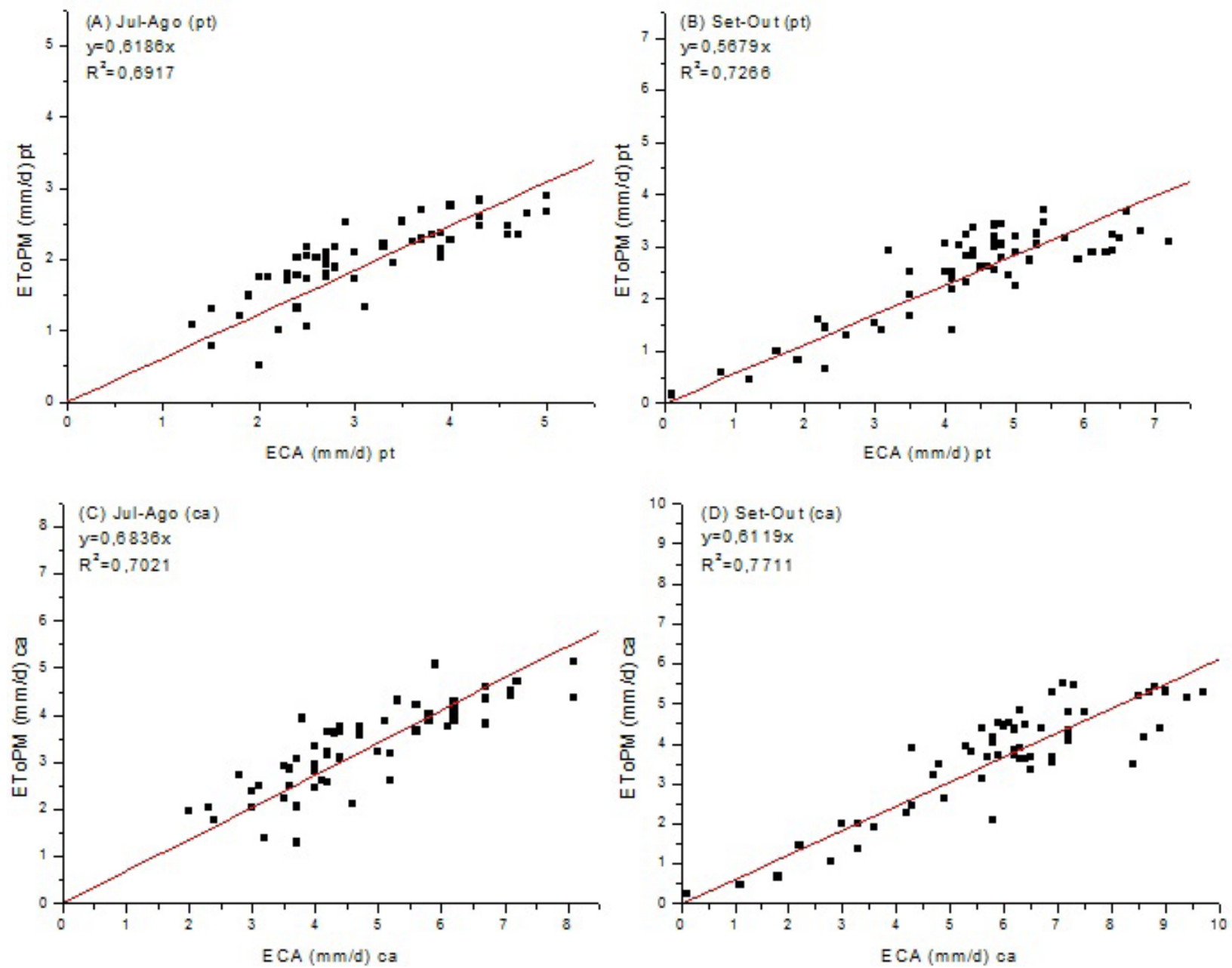

Figura 1. Correlação entre a evapotranspiração de referência por Penman-Monteith (EToPM) e a evaporação do tanque Classe A (ECA) em ambiente protegido para os meses de Jul-Ago (A) e Set-Out (B), e em campo para os meses de Jul-Ago (C) e Set-Out (D). Botucatu, SP. 
A temperatura e umidade relativa do ar diferiram pouco entre os meses de Jul-Ago e Set-Out. Os elementos meteorológicos que mais afetaram a evapotranspiração entre os dois ambientes estudados foram o vento (21 vezes maior nos meses de Jul-Ago e 28 vezes maior nos meses de Set-Out em condição de campo) e o saldo de radiação ( $23 \%$ e $21 \%$ menor no ambiente protegido, respectivamente nos meses de Jul-Ago e Set-Out), aumentando conseqüentemente a evapotranspiração na condição de campo (Tabela 1).

As correlações entre a EToPM (padrão FAO-56) e a evapotranspiração de referência obtida pelo tanque Classe A (EToTCA) a partir do Kp pelos métodos: Doorenbos e Pruitt, Cuenca, Snyder, Pereira, Allen e a correlação entre EToPM e ECA, para os meses de
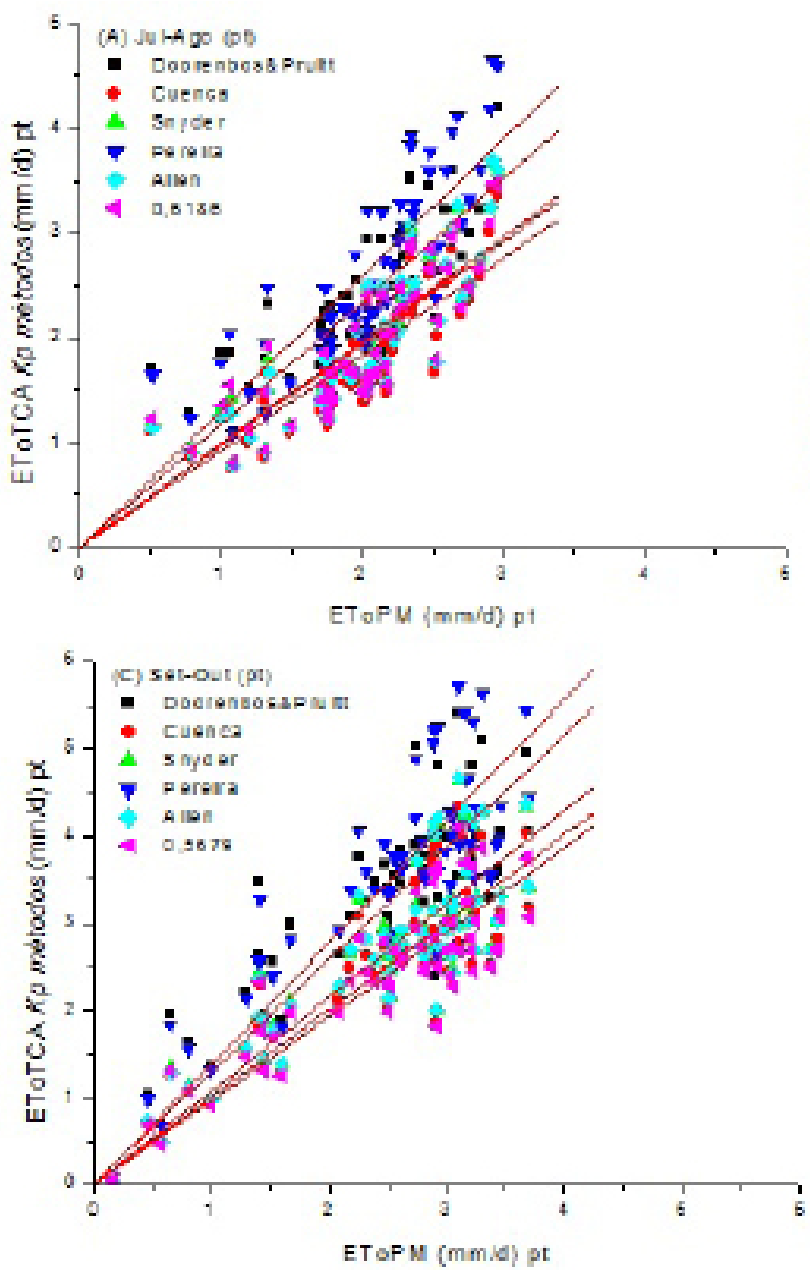

Jul-Ago e Set-Out em ambiente protegido (pt) e campo (ca), são apresentadas na Figura 2.

Comparando a EToTCA determinada pelos diferentes métodos de Kp e a EToPM, no ambiente protegido nos meses de Jul-Ago, os métodos Doorenbos e Pruitt (17,2\%) e Pereira (29,8\%) superestimaram os valores de EToTCA em relação a EToPM, enquanto que os métodos de Cuenca (8\%), Snyder (1,3\%), Allen $(1,6 \%)$ e correlação entre EToPM e ECA $(2,9 \%)$ subestimaram. Nos meses de Set-Out ocorreu superestimativa em relação à EToPM pelos métodos Doorenbos e Pruitt (29,5\%), Cuenca (0,4\%), Snyder (7,6\%), Pereira $(39,6 \%)$ e Allen $(7,5 \%)$, sendo que correlação entre EToPM e ECA $(3,1 \%)$ subestimou (Figura 2 e Tabela 2).
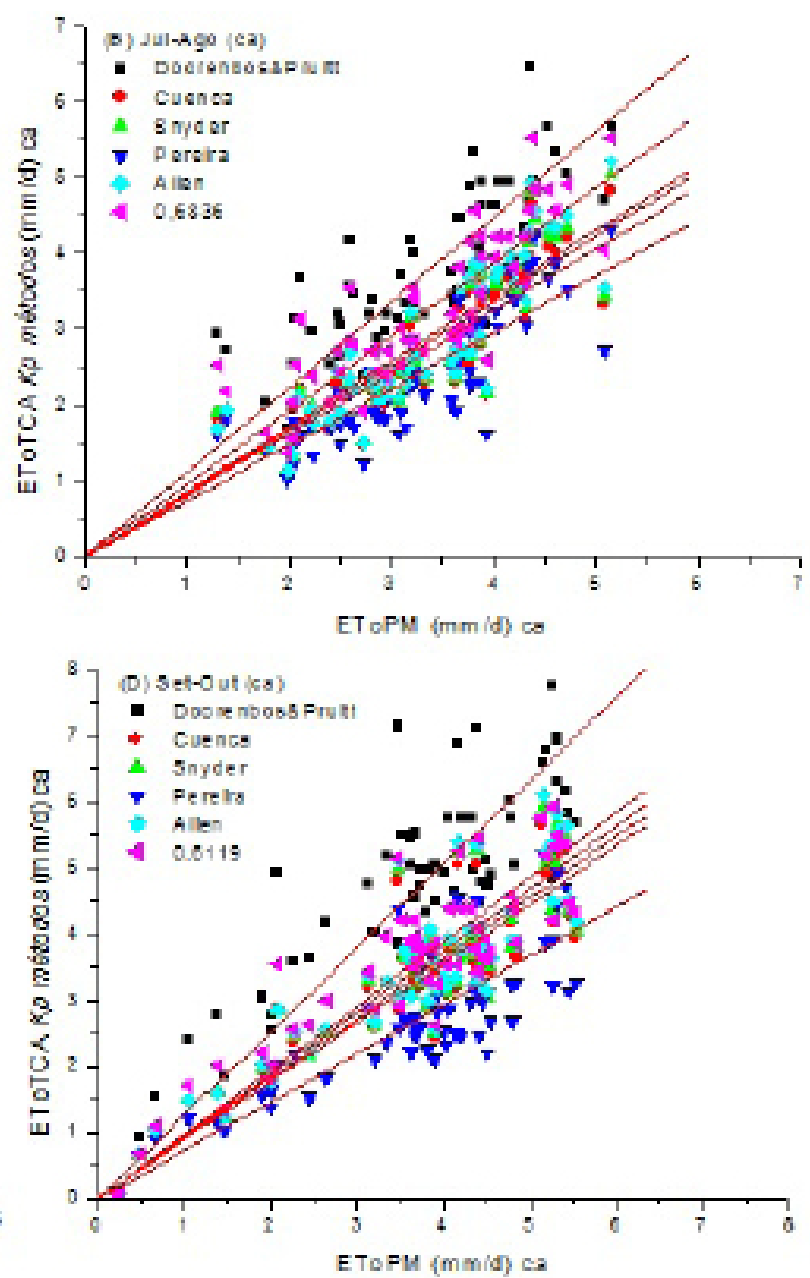

Figura 2. Correlação entre a evapotranspiração de referência por Penman-Monteith (EToPM) e a evapotranspiração de referência pelo tanque Classe A (EToTCA) estimada a partir dos métodos de Kp: Doorenbos e Pruitt, Cuenca, Snyder, Pereira, Allen e a correlação entre EToPM e ECA, para os meses de Jul-Ago e Set-Out em ambiente protegido (pt) e campo (ca). Botucatu, SP. 
Em campo, verificou-se superestimativa apenas pelo método Doorenbos e Pruitt em 12,4\% e $26,7 \%$, respectivamente para os meses de JulAgo e Set-Out. No entanto, nos meses de JulAgo, os métodos que subestimaram a EToTCA em relação a EToPM foram Cuenca $(18,6 \%)$, Snyder $(15,4 \%)$, Pereira $(25,8 \%)$, Allen $(13,7 \%)$ e a correlação entre EToPM e ECA (2,5\%). Já nos meses de Set-Out, os métodos que subestimaram foram Cuenca $(10,8 \%)$, Snyder (8,6\%), Pereira $(27,0 \%)$, Allen $(6,0 \%)$ e a correlação entre EToPM e ECA (2,6\%) (Figura 2 e Tabela 3 ).

Analisando entre os meses, tanto para o ambiente protegido como em campo, verificouse que houve maior dispersão da ETo obtida pelos métodos de Kp nos meses de Set-Out, principalmente para os de Doorenbos e Pruitt e Pereira, comprovado pelo erro médio absoluto (MBE). Entre os ambientes, a condição de campo foi a que apresentou maior dispersão dos dados (Tabelas 2 e 3, e Figura 2).

Assim, em ordem decrescente segundo os indicadores estatísticos, coeficiente de determinação $\left(\mathrm{R}^{2}\right)$, índice de concordância (d), erro médio absoluto (MBE), eficiência (EF) e o coeficiente angular resultante da análise de regressão linear simples, os métodos de Kp que melhor estimaram a EToTCA em função da EToPM em ambiente protegido foram: Allen, Snyder, Cuenca, a correlação entre EToPM e ECA, Doorenbos e Pruitt e Pereira, para os meses de Jul-Ago. Nos meses de Set-Out, a ordem foram: a correlação entre EToPM e ECA, Cuenca, Allen, Snyder, Doorenbos e Pruitt e Pereira.

Na condição de campo, em ordem decrescente também, foram: Allen, correlação entre EToPM e ECA, Snyder, Cuenca, Pereira e Doorenbos e Pruitt para os meses de Jul-Ago; enquanto que para os meses de Set-Out foram: Allen, Cuenca, Snyder, correlação entre EToPM e ECA, Pereira e Doorenbos e Pruitt. Os resultados obtidos em campo corrobora com os autores Sentelhas e Folegatti (2003) para a região de Piracicaba, SP, Mendonça et al. (2006) para Campos dos Goytacazes, RJ e Braga et al. (2008) para a região do Vale do Submédio São Francisco, BA. 
Tabela 2. Indicadores estatísticos entre a evapotranspiração de referência por Penman-Monteith (EToPM) e a evapotranspiração de referência pelo tanque Classe A (EToTCA) por diferentes métodos do coeficiente do tanque Classe $A(\mathrm{Kp})$ : coeficiente de determinação $\left(\mathrm{R}^{2}\right)$, índice de concordância (d), erro máximo $(\mathrm{ME})$, erro médio absoluto (MBE) e eficiência (EF). Ambiente protegido. Botucatu, SP.

\begin{tabular}{lcccccc}
\hline Método Kp (Jul-Ago) & $\mathbf{R}^{\mathbf{2}}$ & $\mathbf{d}$ & $\mathbf{M E}(\mathbf{m m} / \mathbf{d})$ & $\mathbf{M B E}(\mathbf{m m} / \mathbf{d})$ & $\mathbf{E F}$ & EToTCA=b(EToPM)(mm/d) \\
\hline Doorenbos e Pruitt & 0,663 & 0,981 & 0,34 & 0,37 & 0,823 & EToTCA=1,1716(EToPM) \\
Cuenca & 0,705 & 0,985 & 0,85 & 0,17 & 0,934 & EToTCA=0,9200(EToPM) \\
Snyder & 0,706 & 0,988 & 0,72 & 0,04 & 0,938 & EToTCA=0,9871(EToPM) \\
Pereira & 0,703 & 0,973 & 0,14 & 0,59 & 0,774 & EToTCA=1,2983(EToPM) \\
Allen & 0,718 & 0,988 & 0,74 & 0,05 & 0,94 & EToTCA=0,9841(EToPM) \\
Kp=0,6186 & 0,692 & 0,987 & 0,72 & 0,06 & 0,872 & EToTCA=0,9706(EToPM) \\
Método Kp (Set-Out) & & & & & & \\
Doorenbos e Pruitt & 0,694 & 0,962 & 0,51 & 0,82 & 0,692 & EToTCA=1,2947(EToPM) \\
Cuenca & 0,715 & 0,984 & 1,04 & 0,04 & 0,934 & EToTCA=1,0036(EToPM) \\
Snyder & 0,716 & 0,983 & 0,90 & 0,23 & 0,913 & EToTCA=1,0758(EToPM) \\
Pereira & 0,729 & 0,956 & 0,28 & 1,05 & 0,658 & EToTCA=1,3957(EToPM) \\
Allen & 0,713 & 0,983 & 0,90 & 0,22 & 0,918 & EToTCA=1,0754(EToPM) \\
Kp=0,5679 & 0,852 & 0,985 & 1,10 & 0,04 & 0,936 & EToTCA=0,9688(EToPM) \\
\hline
\end{tabular}

*Dados medidos no período de 01/07/99 à 31/10/99 obtidos na área experimental próxima (200 m) à estação meteorológica do Departamento de Recursos Naturais da Faculdade de Ciências Agronômicas da Universidade Estadual Paulista, Campus de Botucatu, SP.

Tabela 3. Indicadores estatísticos entre a evapotranspiração de referência por Penman-Monteith (EToPM) e a evapotranspiração de referência pelo tanque Classe A (EToTCA) por diferentes métodos do coeficiente do tanque Classe $A(\mathrm{Kp})$ : coeficiente de determinação $\left(\mathrm{R}^{2}\right)$, índice de concordância (d), erro máximo (ME), erro médio absoluto (MBE) e eficiência (EF). Campo. Botucatu, SP.

\begin{tabular}{lcccccc}
\hline Método Kp (Jul-Ago) & $\mathbf{R}^{2}$ & $\mathbf{d}$ & $\mathbf{M E}(\mathbf{m m} / \mathbf{d})$ & $\mathbf{M B E}(\mathbf{m m} / \mathbf{d})$ & $\mathbf{E F}$ & $\mathbf{E T o T C A}=\mathbf{b}(\mathbf{E T o P M})(\mathbf{m m} / \mathbf{d})$ \\
\hline Doorenbos e Pruitt & 0,652 & 0,985 & 0,89 & 0,47 & 0,899 & EToTCA=1,1244(EToPM) \\
Cuenca & 0,746 & 0,979 & 1,82 & 0,62 & 0,940 & EToTCA=0,8139(EToPM) \\
Snyder & 0,747 & 0,983 & 1,78 & 0,51 & 0,948 & EToTCA=0,8456(EToPM) \\
Pereira & 0,696 & 0,964 & 2,34 & 0,86 & 0,906 & EToTCA=0,7419(EToPM) \\
Allen & 0,764 & 0,984 & 1,70 & 0,47 & 0,953 & EToTCA=0,8631(EToPM) \\
Kp=0,6836 & 0,702 & 0,990 & 1,33 & 0,06 & 0,952 & EToTCA=0,9749(EToPM) \\
Método Kp (Set-Out) & & & & & & \\
Doorenbos e Pruitt & 0,724 & 0,963 & 0,46 & 1,10 & 0,737 & EToTCA=1,2666(EToPM) \\
Cuenca & 0,786 & 0,982 & 1,57 & 0,36 & 0,950 & EToTCA=0,8915(EToPM) \\
Snyder & 0,779 & 0,983 & 1,45 & 0,27 & 0,949 & EToTCA=0,9141(EToPM) \\
Pereira & 0,676 & 0,959 & 2,31 & 0,94 & 0,897 & EToTCA=0,7300(EToPM) \\
Allen & 0,784 & 0,984 & 1,35 & 0,18 & 0,951 & EToTCA=0,9402(EToPM) \\
Kp=0,6119 & 0,771 & 0,984 & 1,26 & 0,03 & 0,938 & EToTCA=0,9735(EToPM)
\end{tabular}

*Dados medidos no período de 01/07/99 à 31/10/99 obtidos na área experimental próxima (200 m) à estação meteorológica do Departamento de Recursos Naturais da Faculdade de Ciências Agronômicas da Universidade Estadual Paulista, Campus de Botucatu, SP. 
$\mathrm{Na}$ (Figura 3) é apresentada a faixa de Kp de cada método, demonstrando a variabilidade de cada um na estimativa da ETo a partir do método TCA em relação ao calculado pela relação EToPM/ECA, para as condições de ambiente protegido e campo.

As variações do $\mathrm{Kp}$ em ambiente protegido foram menores na maioria dos métodos de Kp utilizados, com exceção dos métodos de Doorenbos e Pruitt e da correlação entre EToPM e ECA, o que contribuiu para uma menor variação do MBE em ambiente protegido em relação ao campo. As variações no ambiente protegido ocorreram desde 0,51 até 0,85 , enquanto que em campo a faixa de variação foi maior, indo desde 0,33 até 0,85 (Figura 3). O fator meteorológico que os métodos de Kp levam em conta, que mais contribuiu para esta variação, foi a velocidade do vento, bem maior na condição de campo, responsável pelo aumento da demanda evaporativa nesta condição, aumentando conseqüentemente a ETo independente do método utilizado, o que corrobora com vários autores já citados na introdução.

Para contemplar mais fielmente as variações microclimáticas em ambiente protegido, recomenda-se utilizar uma série mais longa de dados para o cálculo de $\mathrm{Kp}$, de forma a analisar mensalmente e sazonalmente.

Em condições de campo, Lima e Moura (2003) obtiveram valores de $\mathrm{Kp}$ variando entre 0,54 e 0,81, para o período de junho a dezembro, em Teresina, PI, com um Kp igual a 0,70 dado pela correlação entre ETo Lisímetro e ECA. Em Lages, SC, Cardoso, Ullmann e Eberhardt (2003), encontraram um valor de Kp igual a 0,89 para a correlação entre ETo Lisímetro e ECA, com variação entre 0,45 e 1,19. Braga et al. (2008) recomenda o valor de $\mathrm{Kp}$ igual a 0,55 para todo o ano para a região do Vale do Submédio São Francisco, BA. 

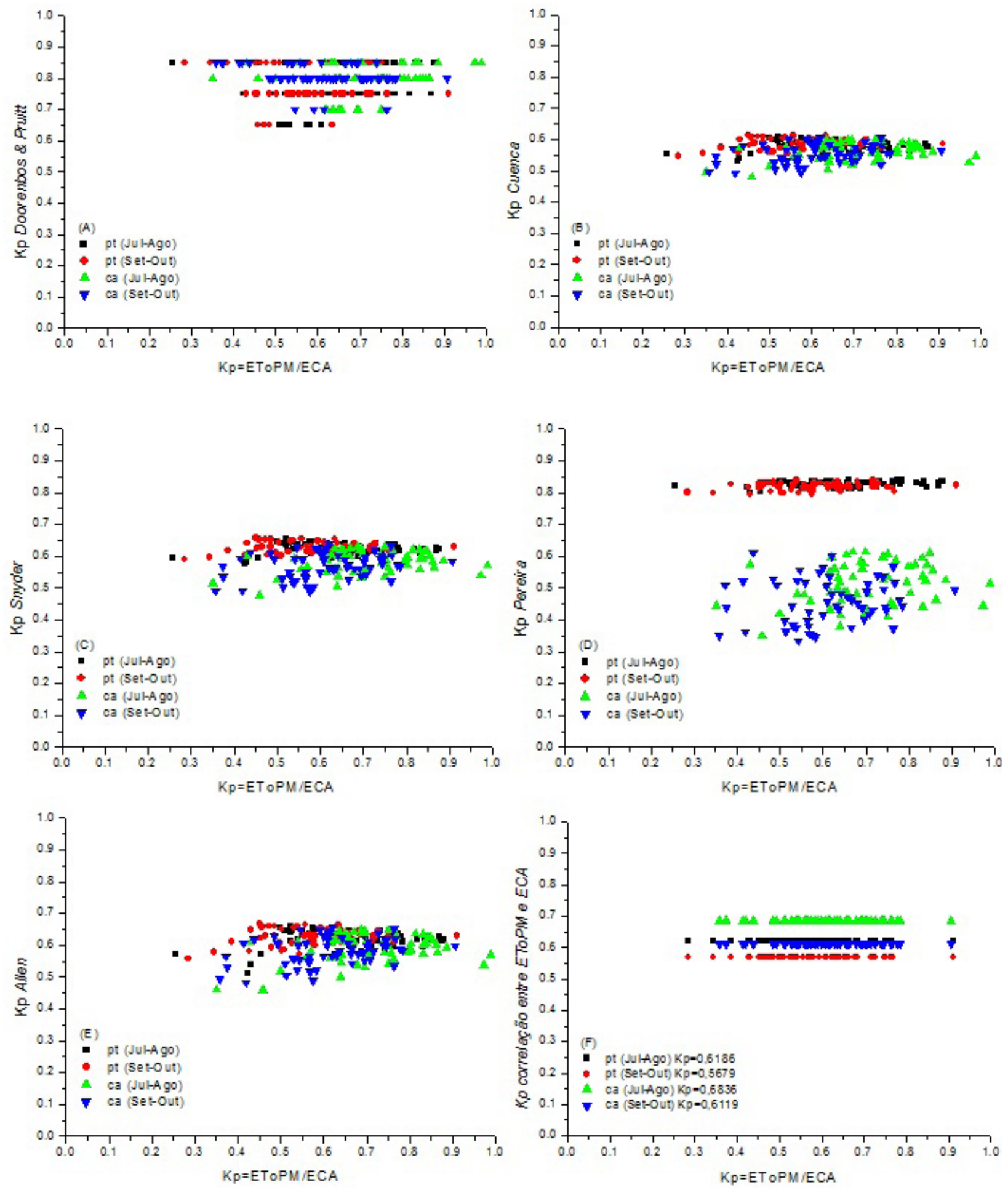

Figura 3. Relação entre valores do coeficiente do tanque Classe $\mathrm{A}(\mathrm{Kp})$ calculados pela razão EToPM/ECA e valores de Kp obtidos pelos métodos: (A) Doorenbos e Pruitt, (B) Cuenca, (C) Snyder, (D) Pereira, (E) Allen e (F) correlação entre EToPM e ECA, em ambiente protegido (pt) e campo (ca). Botucatu, SP. 


\section{Conclusões}

Para ambiente protegido, os métodos de Kp recomendados são os de Allen e Snyder para meses secos, e os da correlação entre EToPM e ECA e Cuenca para meses chuvosos;

Em condição de campo, os métodos de Kp recomendados são os de Allen e da correlação entre EToPM e ECA para meses secos, e os de Allen e Cuenca para meses chuvosos;

O método de Allen foi o mais eficiente na obtenção do Kp, independente do ambiente e dos meses analisados;

$\mathrm{O}$ valor fixo de Kp só deve se utilizado quando não houver a possibilidade de sua determinação a partir de métodos mais sofisticados.

\section{Referências}

ALLEN, R. G.; PEREIRA, L. S.; RAES, D.; SMITH, M. Crop evapotranspiration: guidelines for computing crop water requirements. Roma: FAO, 1998. 300 p. (Irrigation and Drainage, n.56)

ALVES, D. R. B.; KLAR, A. E. Comparação de métodos para estimar evapotranspiração de referência em túnel de plástico. Irriga, Botucatu, v. 1, n. 2, p. 26-34, 1996.

BRAGA, M. B.; CALGARO, M.; MOURA, M. S. B.; SILVA, T. G. F. Coeficientes do tanque classe "A" para estimativa da evapotranspiração de referência na região do Vale do Submédio São Francisco, estado da Bahia. Revista Brasileira de Agrometeorologia, Viçosa, v. 16, n. 1, p. 49-57, 2008.

BRAGA, M. B.; KLAR, A. E. Evaporação e evapotranspiração de referência em campo e estufa orientadas nos sentidos norte-sul e leste-oeste. Irriga, Botucatu, v. 5, n. 3, p. 222-228, 2000.

BURIOL, G. A.; LUZZA, J.; HELDWEIN, A. B.; STRECK, N. A. Evaporação d'água em estufas plásticas e sua relação com o ambiente externo: 1 - avaliação com o uso do tanque classe A e do evaporímetro de Piche. Revista Brasileira de Agrometeorologia, Santa Maria, v. 9, n. 1, p. 35-41, 2001.

CARDOSO, C. O.; ULLMANN, M. N.; EBERHARDT, E. L. Obtenção do coeficiente do tanque evaporimétrico para estimativa da evapotranspiração para LagesSC. In: CONGRESSO BRASILEIRO DE
AGROMETEOROLOGIA, 13., 2003. Santa Maria: Anais... Santa Maria, RS: Sociedade Brasileira de Agrometeorologia, 2003. CD-ROM.

CHIEW, F. H. S.; KAMALADASA, N. N.; MALANO, H. M. Penman-Monteith, FAO-24 reference crop evapotranspiration and class-A pan data in Australia. Agricultural Water Management, Amsterdam, v. 28, n. 1, p. 9-21, 1995.

CUENCA, R. H. Irrigation system design: an engineering approach. New Jersey: Prentice-Hall, Englewood Cliffis. 1989. $133 \mathrm{p}$.

CUNHA, A. R. Parâmetros agrometeorológicos de cultura de pimentão (capsicum annuum l.) em ambientes protegido e campo. 2001. Tese (Doutorado em Energia na Agricultura) - Faculdade de Ciências Agronômicas da UNESP. Campus de Botucatu, Botucatu.

CUNHA, A. R.; ESCOBEDO, J. F.; KLOSOWSKI, E. S. Estimativa do fluxo de calor latente pelo balanço de energia em cultivo protegido de pimentão. Pesquisa Agropecuária Brasileira, Brasília, v. 37, n. 6, p. 735-743, 2002.

CUNHA, A. R.; MARTINS, D. Classificação climática para os municípios de Botucatu e São Manuel, SP. Irriga, Botucatu, v. 14, n. 1, p. 1-11, 2009.

DOORENBOS, J.; PRUITT, W. O. Crop water requirements. Rome: FAO, 1977. 179 p. (FAO. Irrigation and Drainage Paper, 24).

FARIAS, J. R. B. Respostas do feijão-vagem a disponibilidade hídrica associada a alterações micrometeorológicas em estufa plástica. 1991. Tese (Doutorado em Agronomia) - Faculdade de Agronomia, UFRGS, Porto Alegre.

FARIAS, J. R. B.; BERGAMASCHI, H.; MARTINS, S. R. Evapotranspiração no interior de estufas plásticas. Revista Brasileira de Agrometeorologia, Santa Maria, v. 2, n. 1, p. 17-22, 1994.

FERNANDES, C.; CORÁ, J. E.; ARAÚJO, J. A. C. Utilização do tanque classe a para a estimativa da evapotranspiração de referência dentro de casa de vegetação. Engenharia Agrícola, Jaboticabal, v. 24, n. 1, p. 46-50, 2004.

FERNANDEZ, M. D.; ORGAZ, F.; LOPEZ-GÁLVEZ, J. La demanda evaporativa en el invernadero Parral. In: SIMPOSIUM IBEROAMERICANUM SOBRE APLICACION DE LOS PLÁSTICOS EN LAS TECNOLOGIAS AGRÁRIAS, 1., 1995, Almeria, Resumes... Almeria : INIA, 1995. p. 231-238.

LIMA, M.G.; MOURA, G. C. Coeficiente de tanque Classe A e evapotranspiração de referência medida e estimada 
pelo método de Penman-Monteith. In: CONGRESSO BRASILEIRO DE AGROMETEOROLOGIA, 13., 2003. Santa Maria: Anais... Santa Maria: Sociedade Brasileira de Agrometeorologia, 2003. CD-ROM.

MARTINS, S. R.; FERNANDES, H. S.; ASSIS, F. N.; MENDEZ, M. E. G. Caracterização climática e manejo de ambientes protegidos: a experiência brasileira. Informe Agropecuário, Belo Horizonte, v. 20, n. 200/201, p. 15-23, 1999.

MARTINS, S. R.; GONZALEZ, J. F. Evapotranspiração e respostas fisiológicas do feijão-vagem cultivado em substrato em estufa com sistema de ventilação/calefação. Revista Brasileira de Agrometeorologia, Santa Maria, v. 3, n. 1, p. 31-37, 1995.

MENDONÇA, J. C.; SOUSA, E. F.; ANDRÉ, R. G. B.; BERNARDO, S. Coeficientes do tanque Classe A para estimativa da evapotranspiração de referência em Campos dos Goytacazes, RJ. Revista Brasileira de Agrometeorologia, Piracicaba, v. 14, n. 1, p. 123-128, 2006.

MONTERO, J. I.; CASTILLA, N.; GUTIERREZ de RAVÉ, E.; BRETONES, F. Climate under plastic in the Almeria area. Acta Horticulturae, Wageningen, v. 170, n. 1, p. 227-234, 1985.

PEREIRA, A. R.; VILLA NOVA, N.; PEREIRA, A. S.; BARBIERI, V. A model for the class A pan coefficient. Agricultural and Forest Meteorology, Amsterdam, v. 76, n. 2, p. 75-82, 1995.

PRADOS, N. C. Contribuicion al estudio de los cultivos enarenados en Almeria: necessidades hídricas y extracion de los nutrientes del cultivo de tomate de crescimento indeterminado en abrigo de polietileno. 1986. Tese (Doutorado em Fitotecnia) - Cajá Rural Provincial, Almeria, Espanha.

REZENDE, F. C.; ALVES, D. R. B.; FURLAN, R. A.; PASSOS, K. S.; FRIZZONE, J. A.; FOLEGATTI, M.V. Determinação da evaporação em casa de vegetação utilizando tanque reduzido e atmômetro. Irriga, Botucatu, v. 9, n. 3, p. 282-288, 2004.

ROSENBERG, N. J.; McKENNY, M. S.; MARTIN, P. Evapotranspiration in greenhouse-warmed world: a review and a simulation. Agriculture and Forest Meteorology, Amsterdam, v. 47, n. 2/4, p. 303-320, 1989.

SENTELHAS, P. C.; FOLEGATTI, M. V. Class-A pan coefficients $(\mathrm{Kp})$ to estimate daily reference evapotranspiration (ETo). Revista Brasileira de Engenharia Agrícola e Ambiental, Campina Grande, v. 7, n. 10, p. 111-115, 2003.

SENTELHAS, P. C. Agrometeorologia aplicada à irrigação. In: MIRANDA, J. H.; PIRES, R. C. M. (Org.). Irrigação. Piracicaba: SBEA/FUNEP, 2001, v. 1, p. 63120.

SNYDER, R. L. Equation for evaporation pan to evapotranspiration conversion. Journal of Irrigation and Drainage Enginnering of ASCE, New York, v. 118, n. 6, p. 977-980, 1992.

STAMATO JUNIOR, R. P. Condições meteorológicas e consumo de água por mudas cítricas em ambiente protegido. 2007. Dissertação (Mestrado em Agricultura Tropical e Subtropical) - Instituto Agronômico de Campinas, Campinas.

STANGHELLINI, C. Evapotranspiration in greenhouses with especial reference to Mediterranean conditions. Acta Horticulturae, Almeria, v. 1, n. 335, p. 295-304, 1993.

VAN DER POST, C. J.; VAN-SHIE, J. J.; GRAAF, R. Basic problems of water relationship: energy balance and water suply in glasshouses the West-Nertherlands. Acta Horticulturae, Geisenheim, v. 1, n. 35, p. 13-21, 1974.

VÁSQUEZ, M. A. N.; FOLEGATTI, M. V.; DIAS, N. S.; SILVA, C. R. Efeito do ambiente protegido cultivado com melão sobre os elementos meteorológicos e sua relação com as condições externas. Engenharia Agrícola, Jaboticabal, v. 25, n. 1, p. 137-143, 2005.

WILLMOTT, C. J.; ACKLESON, S. G.; DAVIS, R. E.; FEDDEMA, J. J.; KLINK, K. M.; LEGATES, D. R.; O'DONNELL, J.; ROWE, C. M. Statistics for the evaluation and comparison of models. Journal of Geophysical Research, Ottawa, v. 90, n. C5, p. 89959005, 1985.

WORLD RESOURCES INSTITUTE - WRI. Freshwater resources. 2005. Disponível em: <http://earthtrends.wri. org/pdf_library/data_tables/food_water_2008.pdf>. Acesso em: 20 jul. 2009. 
Cunha, A. R. 